\title{
Design Analysis of a Fish-Shaped PVDF Compliance Microgripper
}

\author{
Neeta Sahay*, Sanjoy Das** \\ * Department of Applied Electronic \& Instrumentation Engineering, Academy of Technology, West Bengal, India \\ ** Department of Engineering \& Technological Studies, University of Kalyani, West Bengal, India
}

\begin{tabular}{|c|c|}
\hline Article Info & ABSTRACT \\
\hline Article history: & In this study the design and analysis of compliance microgripper made of \\
\hline Received Feb 10, 2016 & inverse piezoelectric effect has been used in which the required amount of \\
\hline Revised Apr 28, 2016 & voltage has been applied at specific points at the base of the structure to \\
\hline Accepted May 15, 2016 & $\begin{array}{l}\text { produce the force at the tip and thereby the necessary deflection for gripping. } \\
\text { The simulated results of frequency response, force-voltage relationship and }\end{array}$ \\
\hline Kevword. & \\
\hline
\end{tabular}

Compliant Structure

Microgripper

Piezoelectricity

PVDF

Copyright $@ 2016$ Institute of Advanced Engineering and Science. All rights reserved.

\section{Corresponding Author:}

Neeta Sahay,

Departement of Applied Electronic \& Instrumentation Engineering,

Academy of Technology,

Adisaptagram, West Bengal, India.

Email: neetashy82@gmail.com

\section{INTRODUCTION}

Piezoelectricity is a linear coupling between electrical and mechanical processes. In the direct piezoelectric effect, when a piezoelecctric material is compressed, an electric polarization is formed across the material. Poly vinylidene-fluoride (PVDF) is a piezoelectric polymer, which for actuator applications often come in the form of a thin sheet (30 microns thick).

PVDF is typically a semi-crystalline polymer that is approximately $50 \%$ amorphous. It is most commonly synthesized through the free radical polymerization of 1,1-difluoroethylene. The medium for synthesis is usually water with peroxy compounds acting as the polymerization catalysts. The structure of the monomer is $-\mathrm{CH} 2-\mathrm{CF} 2-$, and the chains occur mostly in a head to tail configuration. The molecular weight of PVDF remains typically between 60 and $70 \mathrm{~kg} / \mathrm{mol}$ [2].

Microgrippers are micro-devices used for manipulation of micro-objects in microassembly and microsurgery. In micromanipulation and microassembly systems, micro-grippers act as end-effectors which come in contact with the manipulated objects directly. Grasping and manipulating small or micro-objects are required for a wide range of applications, such as the assembly of small parts to obtain micro or miniature systems, surgery and research in biology and biotechnology[6]. Compliance design provides simple, singlestructured element and adds less complexity in manufacturing and operation of the micro grippers.

\section{RESPONSE OF PVDF MICROGRIPPER}

\subsection{PVDF material structure}

A key characteristic of these materials is the utilization of the converse piezoelectric effect to actuate the structure in addition to the direct effect to sense structural deformation [3]. PVDF has shown to be 
very useful as a dielectric, because of its high permittivity and dielectric strength and low dissipation factor. Compared to other polymers and other piezoelectric materials, in general, PVDF's $\alpha$-phase occurs in a transgauche-trans-gauche (TGTG) formation [2]. This formation, as seen in Fig.1, is not a helical or a planar zigzag but a combination of the two. Either a series of G or TG would represent a purely helical structure. The G's represent the bonds that skew the carbon backbone from the plane. Each $\mathrm{G}$ or G- bond represents a $60^{\circ}$ or $-60^{\circ}$ angle respectively from the plane of the last bond. The $\beta$-crystal phase of PVDF forms a planar zigzag or TT, where T represents a trans-bond that remains in the same plane as the carbon backbone. The all-trans-structure of $\beta$-phase PVDF forces the fluorine atoms along the carbon backbone to come closer together and overlap their van der Waals radii. Because of the van der Waals forces acting between the atoms along the carbon backbone and between the molecules of the polymer, the $\beta$-phase has actually more intermolecular stability, while the $\alpha$-phase is favoured on an intramolecular basis.

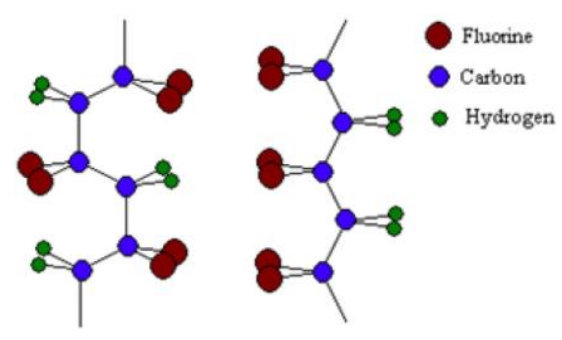

Figure 1. Structure of $\alpha$-PVDF (left) and $\beta$-PVDF (right)

\subsection{PVDF Microgripper Design}

The design for the proposed microgripper using Pro Release 5.0 software has been given in Figure 2

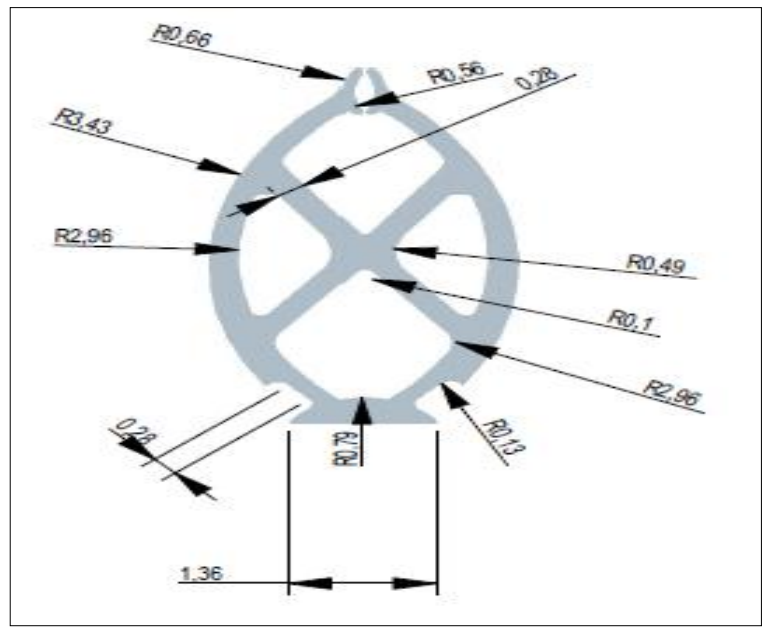

Figure 2a. Design of microgripper (All the dimensions are in $\mathrm{mm}$ (Top view)

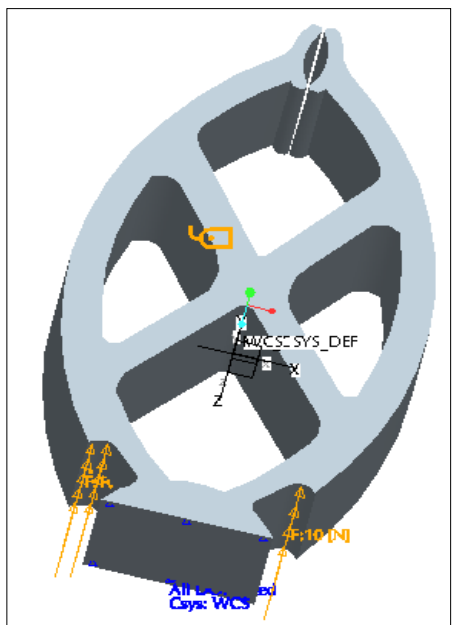

Figure 2b. Design of of microgripper (3D view)

\subsection{Frequency Response}

The voltage output $V(s)$ of a PVDF sensor due to an applied force $F(s)$ in laplace domain can be written as[4][5],

$$
\begin{aligned}
& \frac{V(s)}{F(s)}=\frac{d_{33}}{A \varepsilon_{33}^{T} / h} \frac{\tau s}{(1+\tau s)} \\
& \text { Or, } \frac{V(s)}{F(s)}=\frac{k \tau s}{(1+\tau s)}, \text { in which }
\end{aligned}
$$


$K:$ constant gain of the system $=\frac{d_{33}}{A \varepsilon_{33}^{T} / h}$

$A$ : Area of the crystal plate $\left(\mathrm{m}^{2}\right)$, [herein, the effective area of the fish shaped gripper]

$T$ : Thickness of the plate $(\mathrm{m})$

$\varepsilon_{33}^{T}$ : Mechanical strain in 3-direction due to tensile stress $(T)$ in the 3-direction (which represents the thickness direction) $\left(\mathrm{Fm}^{-1}\right)$ (PVDF normal dielectric constant)

$\tau \quad$ : Time constant of PVDF sensor and

$=\frac{\rho h C_{\mathrm{p}}}{A}=R_{\mathrm{p}} C_{\mathrm{p}} ;$ In which, $R_{\mathrm{p}}=\frac{\rho h}{A}$ represents the resistance of PVDF sensor, $\rho$ and $C_{\mathrm{p}}$ are the resistivity and capacitance of PVDF material measured in $\Omega m$ and F.

$d_{33}$ : PVDF normal piezoelectric constant $\left(\mathrm{CN}^{-1}\right)$

$h$ : distance through which the force to be transmitted (m)

The polymer considered for this study is PVDF-1000 with a thickness $1 \mathrm{~mm}$. Hence, for this study,

$d_{33}=33 \mathrm{pC} / \mathrm{N}=33 \times 10^{-12} \mathrm{C} / \mathrm{N}$

$h \quad=4.83 \mathrm{~mm}=4.83 \times 10^{-6} \mathrm{~m}$

$\varepsilon_{33}^{T}=7.4 \mathrm{~F} / \mathrm{m}$

$A \quad=0.513 \mathrm{~mm}^{2}$ [Computed according to design]

$\rho \quad=2 \times 10^{14} \Omega-\mathrm{cm}=2 \times 10^{12} \Omega-\mathrm{m}$

$k=42 \times 10^{-9} \mathrm{~V} / \mathrm{N}$

$\tau=\frac{\rho h C_{\mathrm{p}}}{A}=\rho \varepsilon, \quad\left[\right.$ as $\left.C_{\mathrm{p}}=\frac{\varepsilon A}{h}\right]=14.8 \times 10^{12} \mathrm{sec}$

From eq-(1) can be written as

$$
\frac{F(s)}{V(s)}=\frac{1+\tau s}{k \tau s}
$$

Frequency range: $10^{-13}$ to $10^{-10} \mathrm{rad} / \mathrm{sec}$.

From frequency response analysis as shown in Fig.3, it is evident that the gain and phase angle is almost constant over the frequency range of $\omega>10^{-11} \mathrm{rad} / \mathrm{sec}$.

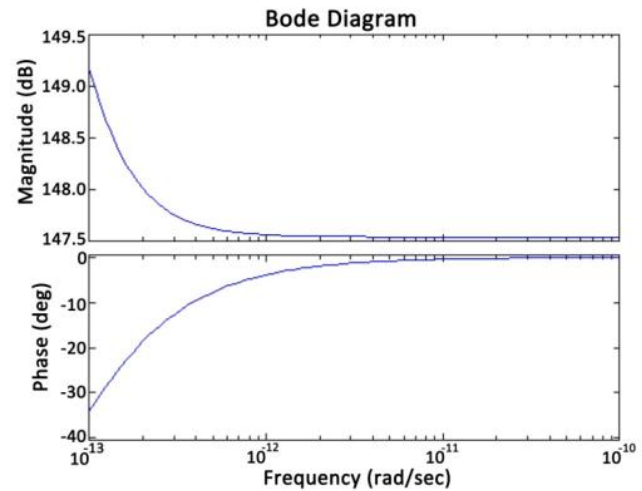

Figure 3. Frequency response of PVDF microgripper

\subsection{Time Response} written as

Time response can be obtained by taking inverse laplace transform of Equation (2), and can be 


$$
f(t)=V\left[23.8 \times 10^{6}+1.6 \times 10^{-6} t\right]
$$

Where, $V$ is the amplitude of the step voltage in Volts. This gives a straight line parallel to time-axis for the time scale up to $10^{6} \mathrm{sec}$.

\section{VOLTAGE-FORCE RELATIONS}

As it is seen from Equation (3) that the variation of force with respect to time is very slow for a constant force, time scale of interest is chosen as 0 to $10 \mathrm{sec}$, for which it is clear from eq.(3) that the generated force $f(t)$ maintains a linear relationship with $V(t)$ as written below:

$$
f(t)=23.8 V(t) N
$$

Varying the voltage from $0 \mu \mathrm{V}$ to $5 \mu \mathrm{V}$ in $0.1 \mu \mathrm{V}$ step, the voltage-force diagram can thus be obtained as shown in Figure 4.

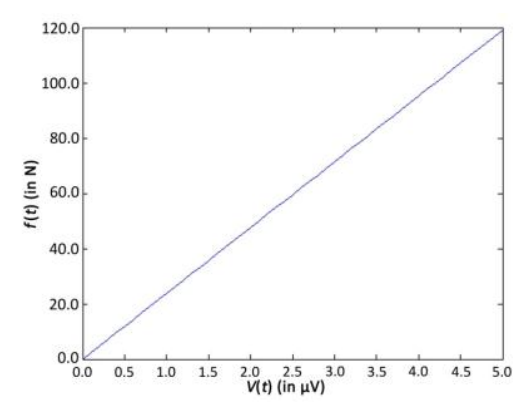

Figure 4. Force Voltage relation of PVDF microgripper

\subsection{Stress and Displacement analysis}

The above design has been analyzed with Creo Element/ Pro Release 5.0 software. The gripper has been subjected to the force of $20 \mathrm{~N}$ in the (-ve) z-direction at the base. The corresponding analysis of stress result and displacement are computed and presented in Fig.5 and Fig.6 respectively.

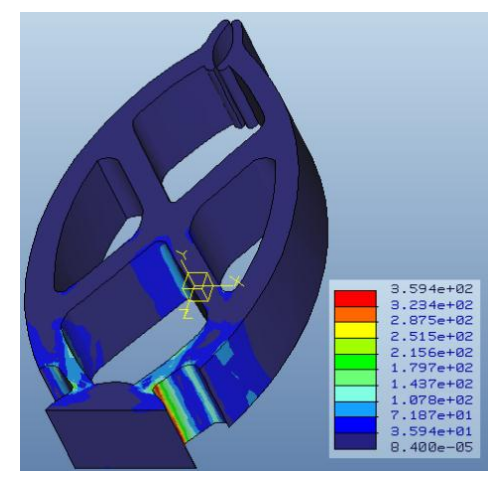

Figure 5. Stress distribution in MPa. Maximum stress has been evaluated as $3.59 \times 10^{2} \mathrm{MPa}$

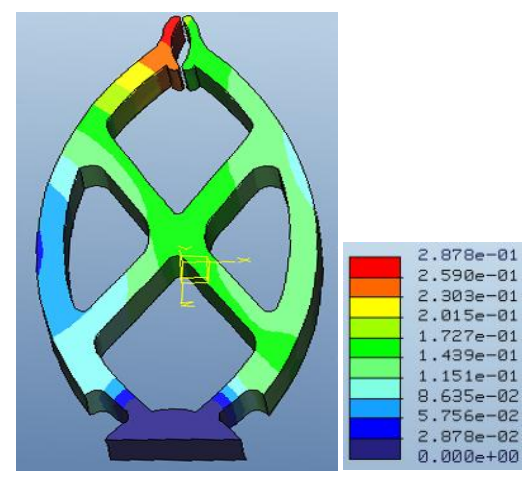

Figure 6. Displacement analysis in mm. Maximum displacement has been evaluated by 0.287 $\mathrm{mm}$

\section{CONCLUSION}

From the above explanations, it can be seen that force-voltage relationship is linear in the range of interest. So if the required value of voltage will be applied at the base of the micro gripper, the voltage will be transmitted over the body and will cause the generation of force at the tip of the gripper resulting opening and closing of the micro gripper. 


\section{REFERENCES}

[1] Laura Marie Lediaev., "Modeling Piezoelectric Pvdf Sheets With Conductive Polymer Electrodes", April 2006.

[2] Daniel M. Esterly., "Manufacturing of Poly (vinylidene fluoride) and Evaluation of its Mechanical Properties", August 9, 2002.

[3] Wenji Aia., Qingsong Xu., "Overview of flexure-based compliant microgrippers", Advances in Robotics Research, Vol. 1, No. 1 (2014) 001-019.

[4] Jayant Sirohi., Inderjit Chopra.," Fundamental Understanding of Piezoelectric Strain Sensors", University of Maryland, College Park, April 2000.

[5] Carmen K. M. Fung., Imad Elhaj ,Wen J. Lil., Ning Xi., “ A 2-D PVDF Force manipulation Sensing System for Micro and Micro-assembly”, Proceedings of the 2002 IEEE.

[6] Philippe Benech., Eric Chamberod., Christian Monllor., "Acceleration Measurement Using PVDF", IEEE Transactions On Ultrasonics, Ferroelectrics, And Frequency Control, Vol. 43, No. 5, September 1996. 